\title{
Experimental Characterization of Ionic Polymer Metal Composite as a Novel Fractional Order Element
}

\author{
Riccardo Caponetto, Salvatore Graziani, Fulvio L. Pappalardo, and Francesca Sapuppo \\ Dipartimento di Ingegneria Elettrica, Elettronica ed Informatica, Università degli Studi di Catania, \\ V. le A. Doria 6, 95125 Catania, Italy \\ Correspondence should be addressed to Riccardo Caponetto; riccardo.caponetto@dieei.unict.it
}

Received 17 May 2013; Accepted 9 June 2013

Academic Editor: Dumitru Baleanu

Copyright (C) 2013 Riccardo Caponetto et al. This is an open access article distributed under the Creative Commons Attribution License, which permits unrestricted use, distribution, and reproduction in any medium, provided the original work is properly cited.

\begin{abstract}
Ionic polymer metal composites (IPMCs) are electroactive materials made of ionic polymer thin membranes with platinum metallization on their surfaces. They are interesting materials due to not only their electromechanical applications as transducers but also to their electrochemical features and the relationship between the ionic/solvent current and the potential field. Their electrochemical properties thus suggest the possibility for exploiting them as compact fractional-order elements (FOEs) with a view of defining fabrication processes and production strategies that assure the desired performances. In this paper, the experimental electrical characterization of a brand new IPMC setup in a fixed sandwich configuration is proposed. Two IPMC devices with different platinum absorption times $(5 \mathrm{~h}$ and $20 \mathrm{~h}$ ) are characterized through experimental data: first, a preliminary linearity study is performed for a fixed input voltage amplitude in order to determine the frequency region where IPMC can be approximated as linear; then, a frequency analysis is carried out in order to identify a coherent fractional-order dynamics in the bode diagrams. Such analyses take the first steps towards a simplified model of IPMC as a compact electronic FOE for which the fractional exponent value depends on fabrication parameters as the absorption time.
\end{abstract}

\section{Introduction}

Ionic polymer-metal composites (IPMCs) as electroactive polymers (EAPs) have the very interesting capabilities of transforming electrical energy into mechanical energy, and vice versa $[1,2]$, making them privileged candidates for the realization of actuators or sensors with features as low required voltage, high compliance, lightness, softness, and so forth, thus, creating great interest in possible applications in very different fields such as robotics, aerospace, and biomedics [3-5].

They are composite materials made of ionic polymers in presence of solvent with layers of noble metals on their surfaces. Their structure and composition make their full exploitation currently limited because of the incomplete knowledge of their working principles and therefore a not clearly defined design procedure.

Due to their electromechanical properties, they have been traditionally characterized as transducers, both as sensors and actuators, and three different strategies have been used to describe the relationship between the electrical and the mechanical behaviors [6]. The first one, referred to as black box and behavioral model, provides a purely empirical model of IPMCs obtained through a series of curve fits based on experimental data [7]. The second approach, called gray box, combines fundamental physical laws with empirically derived parameters to describe IPMCs' electromechanical conversion [6, 8-10]. It provides simplified and reduced models which are also suitable for parameterization. The third level of model is called white box or physical model. It relies on the underlying physical mechanisms of the IPMC to develop a system of distributed equations that fully describe the material response on a multiphysics domain and which are solved though computational methods [11, 12]. In this context, the authors' previous works [13] highlighted the possibility of modeling the IPMC actuators via the gray box model based on fractional-order systems, paving the way for a brand new approach to such materials seen as 
fractional-order electronic elements (FOEs) and not only as a electromechanical transducers.

This new idea is suggested by IPMC electrochemical and structural properties, since the dendrites on the interfacial landscape between the metal electrodes and the polymer layer show fractal dimensions, and a fractional electrical behavior might be due to the anomalous diffusion of ions and solvent through metal/polymer surface [14].

Moreover, IPMCs fit perfectly in the research stream working on FOE implementation processes which assesses the interaction between ionic phenomena and fractal structures as possible bases for FOEs' working principle and realization. After early approaches to FOE implementation by infinite ladder networks [15], research has thus moved from such a bulky circuital solution with a limited range of exponent values to the search for new technological solutions. Dielectric materials as lithium hydrazinium sulfate were studied as a fractional impedance [16], and the influence of temperature on the order of fractional operator has been investigated in [17]. Semiconductor fabrication of FOE was realized via fractal structures on silicon [18] obtaining limited range of exponent values. Other research lines have then exploited composite materials for the implementation of FOEs by developing ionic lithium ions on rough surface of metal electrodes [19]. Also, electrolytic processes have been used with the drawback of electrodes dimension and reproducibility of specifics [20]. More recently, solutions based on polymer-coated probe in polarizable medium have been suggested [21-23]. The main technological points presented in the literature are related to the sizes of the devices, in terms of electrodes or packaging containing the polarizable medium, and the issues in controlling fractional dynamics specifics by fabrication process.

The main goal of this paper is therefore to show the IPMC potential as FOE via experimental characterization.

After describing the IPMC working principles and the manufacturing process, a brand new experimental setup will be presented in Section 2. A brief background on fractionalorder systems and the mathematical tools to work with them is given in Section 3. The main focus about the IPMC experimental characterization as FOE will be then approached in Section 4: first, a preliminary study on linearity is given in order to identify the frequency band where the device can be approximated as linear and therefore to motivate the frequency domain approach. A frequency analysis of two different IPMC devices fabricated with different technological parameters will be at the bases for searching experimental evidence of the fractional-order dynamics of IPMC as FOEs and the possibility of controlling the fractional exponent value by fabrication processes.

\section{IPMC Devices}

2.1. IPMC Structure and Working Principles. IPMCs are based on a polymer containing ions (also called ionomers or ionic polymers) that are weakly linked to the polymer chain and metallized via a chemical process, on both sides, with a noble metal, to realize the electrodes. There are a number of different types of ionic polymers available, but the typical IPMC used in many investigations is composed of a perfluorinated ion-exchange membrane, Nafion 117, which is surface-composited by platinum via chemical process, see Figure 1.

The platinum electrodes often consist of small, interconnected metal particles which are made to penetrate into the ionic polymer membrane. This results in the formation of electrodes with dendritic structures $[1,14]$ which extend from the surface into the membrane.

Working as an actuator, when an external voltage is applied across the thickness of the IPMC, mobile cations $\left(\mathrm{H}_{+}\right)$ in the polymer will move toward the cathode. Moreover, if a solvent is present in the sample, the cations will carry solvent molecules with them. The cathode area will expand, while the anode area will shrink. If the tip of the IPMC strip is free the polymer will bend toward the anode; thus a force will be delivered. On the other hand, when the IPMC works as a sensor, it exploits the mechanical displacement of the polymer for the generation of an ionic current inducing a potential difference.

In general, the relationship between the applied potential and absorbed current will be affected by the ionic current and the solvent flow within the sample and by the interaction of the ions and the solvent molecules with the polymer/metal interface.

2.2. Manufacturing. Nafion 117 films (DuPont, Sigma-Aldrich Group) [24] with thickness $t_{\mathrm{Naf}}=180 \mu \mathrm{m}$ and sizes $4 \mathrm{~cm} \times 4 \mathrm{~cm}$ were pretreated by successive boiling for $30 \mathrm{~min}$ in $\mathrm{HCl}_{2} \mathrm{~N}$ and deionized water. Ethylene glycol (EG) was used as the solvents and platinum as the electrodes. Two platinum metallizations were obtained by immersion of the Nafion 117 membrane in a solution of $\left[\mathrm{Pt}\left(\mathrm{NH}_{3}\right)_{4}\right] \mathrm{Cl}_{2}(\mathrm{MW}$ = 334.12), and immersion time will be here referred to as absorption time. The platinum solution was obtained by dissolving $205 \mathrm{mg}$ of the complex in $60 \mathrm{~mL}$ of deionized water and adding $1 \mathrm{~mL}$ of ammonium hydroxide at $5 \%$. In order to increase the performance of the device, a dispersing agent (polyvinylpyrrolidone with molecular weight 10000-PVP10) has been added. Moreover, a secondary metallization was performed via deposition.

Then, the samples were boiled in $0.1 \mathrm{M} \mathrm{HCl}$ for $1 \mathrm{~h}$. In order to obtain the IPMC with EG as solvent, Nafion 117 membranes were soaked overnight in a beaker containing pure EG and, finally, heated to $60^{\circ} \mathrm{C}$ for $1 \mathrm{~h}$. Obtained IPMC was then cut into strips of size $1 \mathrm{~cm} \times 1 \mathrm{~cm}$ and dried for one week. Some IPMC samples are shown in Figure 2.

Two different IPMC membranes have been fabricated with two different absorption times, $5 \mathrm{~h}$ and $20 \mathrm{~h}$, in order to study the relationship between such a fabrication parameter and the fractional-order dynamics of the IPMC device. The two membranes will be here referred to respectively as IPMC $_{\mathrm{AbsT}-5 \mathrm{~h}}$ and IPMC $\mathrm{AbsT}_{\mathrm{Ab} \mathrm{h} \text {. }}$.

2.3. Geometry and Experimental Setup. The new IPMC device here proposed as FOE consists of an IPMC strip $(1 \mathrm{~cm} \times 1 \mathrm{~cm})$ mechanically fixed within a Plexiglas sandwich 


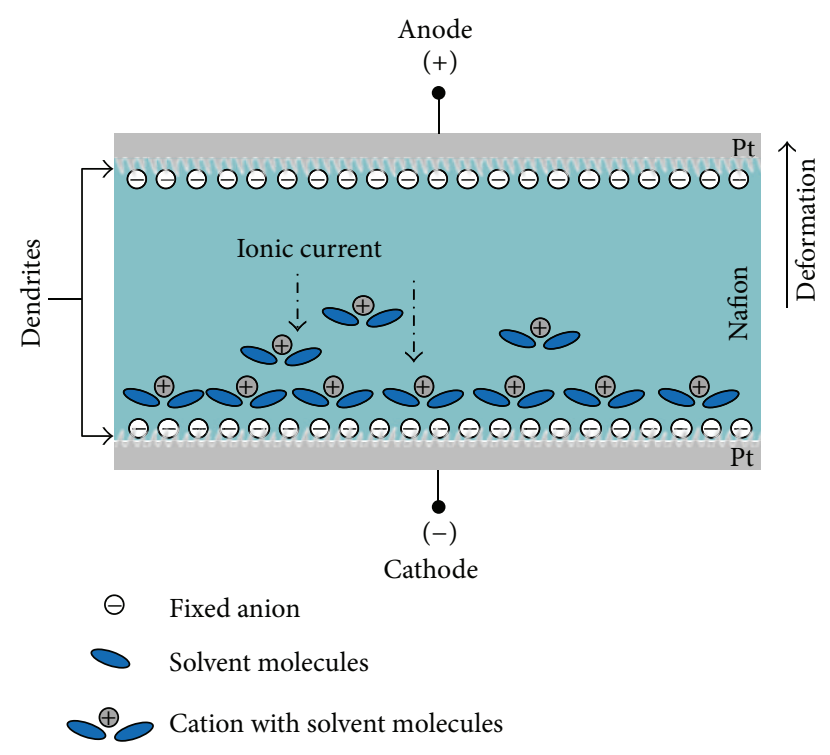

FIGURE 1: Structure and working principle of IPMC.

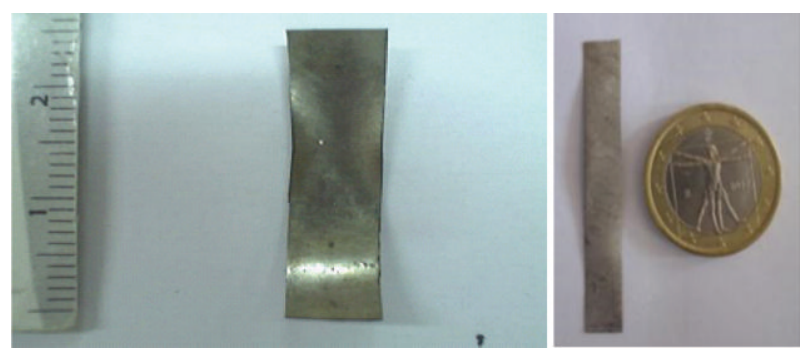

Figure 2: IPMC sample.

configuration in series with a resistor $R=46 \Omega$ as shown in the schematic in Figure 3.

The input voltage signal ( $V_{\text {in }}$ ) was driven by a waveform generator (Agilent 33220A) through a conditioning circuit made of an operational amplifies in buffer configuration (ST TL082CP). The output voltage $\left(V_{\text {out }}\right)$ was measured through a pair of copper electrodes $(1 \mathrm{~cm} \times 1 \mathrm{~cm}$ and thickness $35 \mu \mathrm{m})$ printed on a PBC board and in direct contact with the entire platinum electrodes.

Both the input $V_{\text {in }}$ and output $V_{\text {out }}$ signals were acquired by using a National Instrument (NI USB-6251) board and processed by the LabView software.

A schematic of the experimental setup is reported in Figure 4(a), while details on the IPMC FOE are in Figure 4(b).

\section{Remarks on Fractional Order Systems}

The subject of fractional-order calculus or noninteger-order systems, that is, the calculus of integrals and derivatives of any arbitrary real or complex order, has gained considerable popularity and importance during the last three decades with applications in numerous seemingly diverse and widespread fields of science and engineering [25-27]. The advantages of

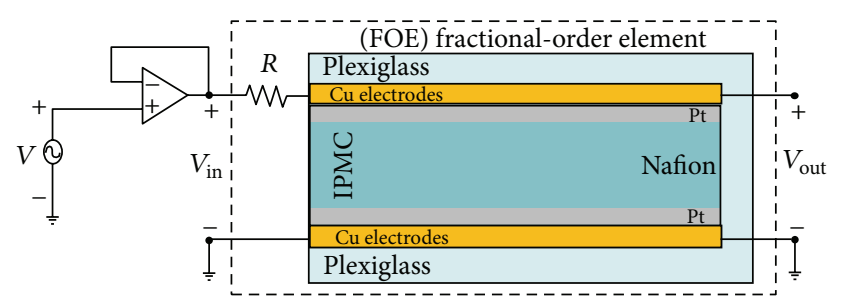

FIGURE 3: IPMC FOE sandwich configuration schematic.

fractional derivatives became apparent in modeling mechanical and electrical properties of real materials.

Fractional derivatives provide an excellent tool for the description of memory and hereditary properties of various materials and processes. This is the main advantage of fractional derivatives in comparison with classical integerorder models, in which such effects are in fact neglected.

Fractional-order system description can be approached applying either constant or variable/distributed fractionalorder models [28]. The aim of this paper is to find a constantorder fractional model in order to define a simplified operator that allows to link fabrication process with FOE parameters.

In this context, the most frequently used definition for the general fractional differintegral is the Caputo one, (see [27]).

$$
{ }_{a} D_{t}^{r} f(t)=\frac{1}{\Gamma(t-n)} \int_{a}^{t} \frac{f^{(n)}(\tau)}{(t-\tau)^{r-n+1}} d \tau
$$

for $(n-1<r<n)$. The initial conditions for the fractionalorder differential equations with the Caputo derivatives are in the same form as for the integer-order differential equations.

In the above definition, $\Gamma(m)$ is the factorial function, defined for positive real $m$, by the following expression:

$$
\Gamma(m)=\int_{0}^{\infty} e^{-u} u^{m-1} d u .
$$

Also for fractional-order systems, it is possible to apply the Laplace transformation. It assumes the following form:

$$
L\left\{\frac{d^{q} f(t)}{d t^{q}}\right\}=s^{q} L\{f(t)\}-\sum_{k=0}^{n-1} s^{k}\left[\frac{d^{q-1-k} f(t)}{d t^{q-k-1}}\right]_{t=0} .
$$

And it allows to easily manage fractional differential equation as noninteger order transfer function.

The fractional-order transfer function of incommensurate real orders assumes the following form [25]:

$$
G(s)=\frac{b_{m} s^{\beta_{m}}+\cdots+b_{1} s^{\beta_{1}}+b_{0} s^{\beta_{0}}}{a_{n} s^{\alpha_{n}}+\cdots+a_{1} s^{\alpha_{1}}+a_{0} s^{\alpha_{0}}},
$$

where $a_{k}(k=0, \ldots n), b_{k}(k=0, \ldots m)$ are constants and $\alpha_{k}(k=0, \ldots n), \beta_{k}(k=0, \ldots m)$ are arbitrary real or rational numbers, and, without loss of generality, they can be arranged as $\alpha_{n}>\alpha_{n-1}>\cdots>\alpha_{0}$ and $\beta_{m}>\beta_{m-1}>\cdots>\beta_{0}$.

Since in this case the values of fractional exponents need to be estimated along with the corresponding transfer function coefficients, the identification problem is nonconvex, and an adequate optimization procedure needs to be used. 


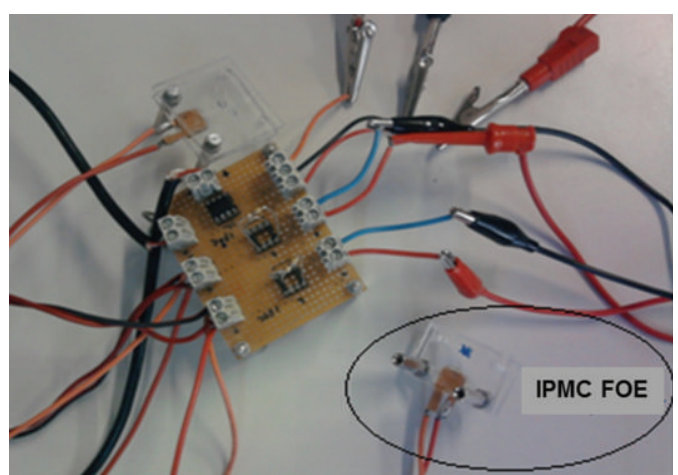

(a)

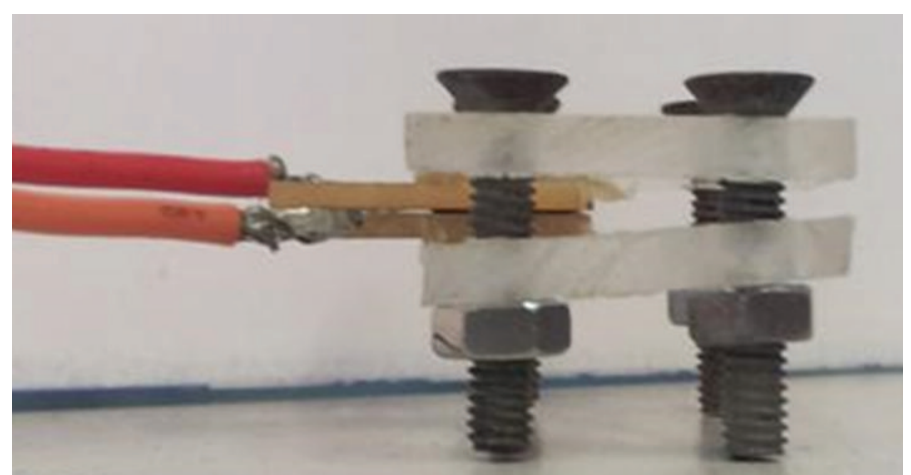

(b)

FIGURE 4: (a) Experimental setup. (b) IPMC FOE setup.
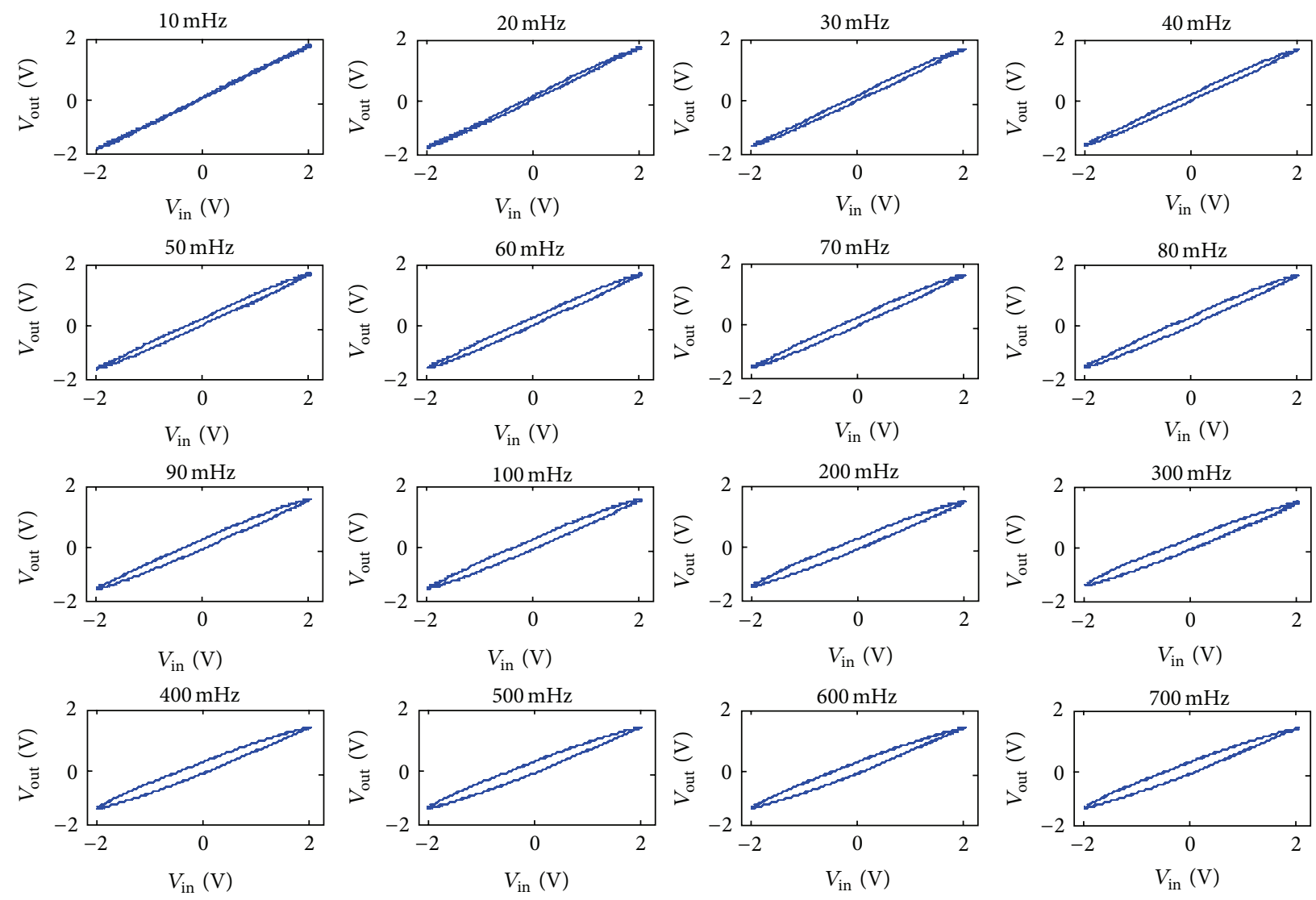

FIGURE 5: Lissajous curves on measured input $\left(V_{\text {in }}\right)$ and output $\left(V_{\text {out }}\right)$ voltages in the frequency range between $10 \mathrm{mHz}$ and $700 \mathrm{mHz}$ for the device IPMC $\mathrm{AbsT}-5 \mathrm{~h}_{\text {. }}$.

\section{IPMC Fractional-Order Element Characterization}

A preliminary linearity analysis based on experimental data has been performed in order to determine the frequency band where the IPMC device can be approximated as linear. Under the linearity hypothesis, a frequency domain characterization approach is proposed here. It has been based on the Bode diagrams (modules and phase) of the transfer function obtained by the ratio between the output $\left(V_{\text {out }}\right)$ and the input $\left(V_{\text {in }}\right)$ voltages in the frequency domain:

$$
G_{\text {IPMC }}(s)=\frac{V_{\text {out }}(s)}{V_{\text {in }}(s)} .
$$

4.1. Experimental Data. Measurements have been performed on the two membranes IPMC $\mathrm{AbsT}-5 \mathrm{~h}_{\mathrm{h}}$ and IPMC $\mathrm{AbsT}-20 \mathrm{~h}_{\mathrm{h}}$. A set 

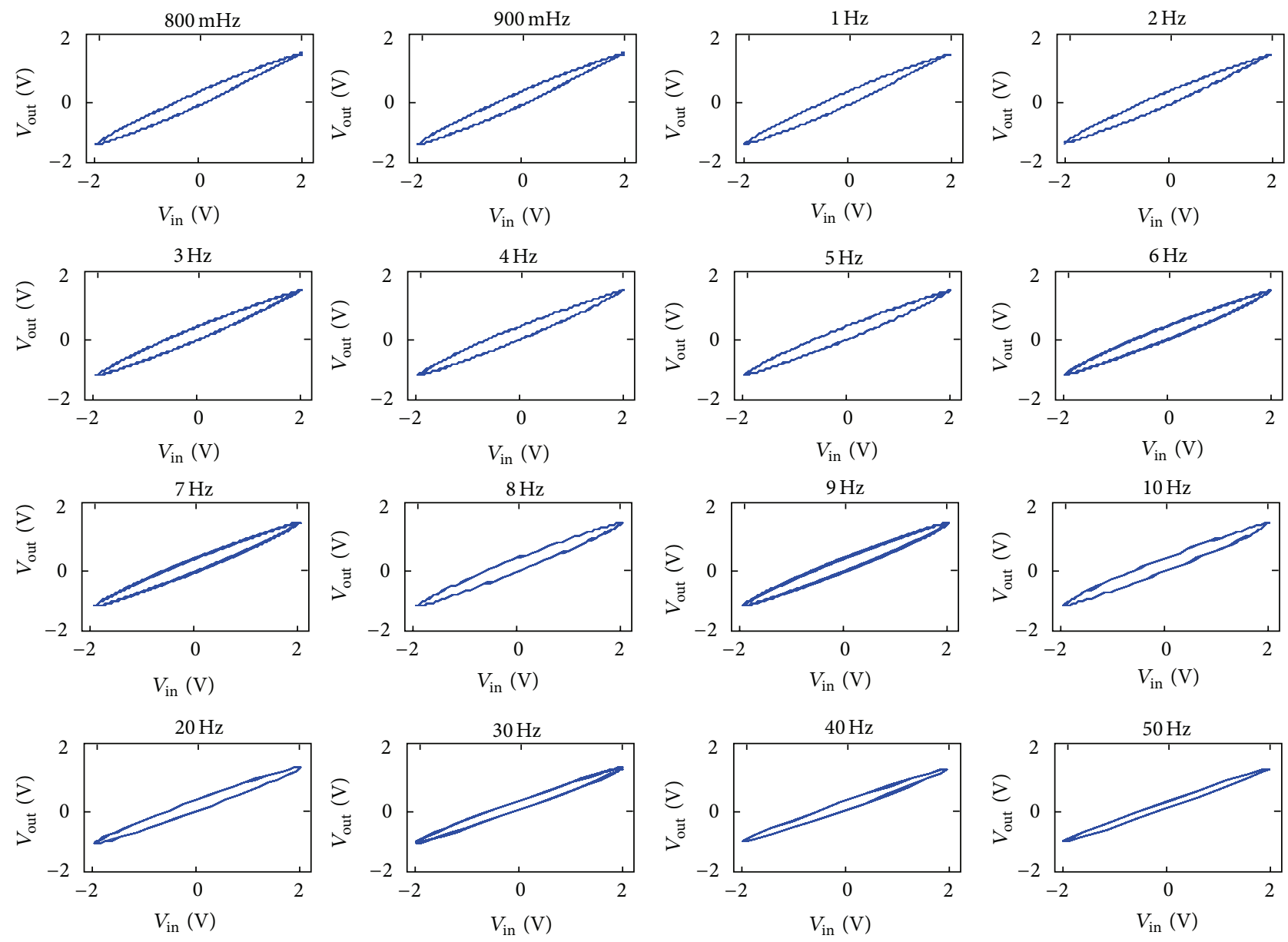

Figure 6: Lissajous curves on measured input $\left(V_{\text {in }}\right)$ and output $\left(V_{\text {out }}\right)$ voltages in the frequency range between $800 \mathrm{mHz}$ and $50 \mathrm{~Hz}$ for the device IPMC $\mathrm{AbsT}_{\mathrm{Ab}} \mathrm{h}$.

of sine voltages with amplitude of $4 V_{\text {pp }}$ was applied as $V_{\text {in }}$, and the output voltage $V_{\text {out }}$ was measured.

For each membrane, different measures were performer varying $V_{\text {in }}$ frequency in the range from $10 \mathrm{mHz}$ to $10 \mathrm{kHz}$ with $10 \mathrm{~Hz}$ step. MATLAB tools were then used to estimate the modulus and phase of the acquired signals.

4.2. Linearity Study. As the literature has shown how IPMC membranes working as transducers present a nonlinear component in the electromechanical model and show a hysteretic behavior in the relationship between applied voltage and absorbed current [8]; the hypothesis of linearity must be verified in this work in order to consider the frequency response as a coherent characterization for IPMC. Moreover, being the system nonlinear, such characterization is valid only for the given input voltage amplitude $\left(4 V_{\mathrm{PP}}\right)$.

Lissajous curves have been therefore studied in order to characterize the linearity of the IPMC as FOE. A Lissajous curve was obtained at each frequency for the complete experimental range for both IPMC $\mathrm{AbsT}_{\mathrm{A}-5 \mathrm{~h}}$ and $\mathrm{IPMC}_{\mathrm{AbsT}-20 \mathrm{~h}}$. Figures 5, 6, 7, and 8 show such curves for the device $\mathrm{IPMC}_{\mathrm{AbsT}-5 \mathrm{~h}}$. Moreover, Figure 9 shows zoomed curves for sample frequencies: $f=50 \mathrm{mHz}, f=1 \mathrm{~Hz}, f=9 \mathrm{~Hz}$, and $f=$ $4 \mathrm{kHz}$.

It is worth noticing that at low frequencies, the nonlinear component dominates, and the Lissajous curves have a nonelliptic shape. In particular, under the $1 \mathrm{~Hz}$ frequency, the curves show nonlinearity, while $1 \mathrm{~Hz}$ is a frequency of transition from nonlinearity to linearity. For frequencies higher than $1 \mathrm{~Hz}$, the Lissajous curves' shapes can be considered elliptic.

The IPMC $\mathrm{AbsT}_{-20 \mathrm{~h}}$ shows the same trend in the Lissajous curves; therefore, the conclusion about IPMC ${ }_{\mathrm{AbsT}-5 \mathrm{~h}}$ linearity will be extended to it.

Such considerations are confirmed by the literature [8], where the IPMC model, as transducer, is represented by a nonlinear component connected to capacitive elements. At low frequencies, the capacitances are considered open circuits and the nonlinearity dominates the global behavior. As the frequency increases, the linear capacitive effect of IPMC becomes significant and dominant with respect to the nonlinear component.

Concluding, in this work, IPMC FOE will be approximated as linear in a frequency range from $1 \mathrm{~Hz}$ to $10 \mathrm{kHz}$. 

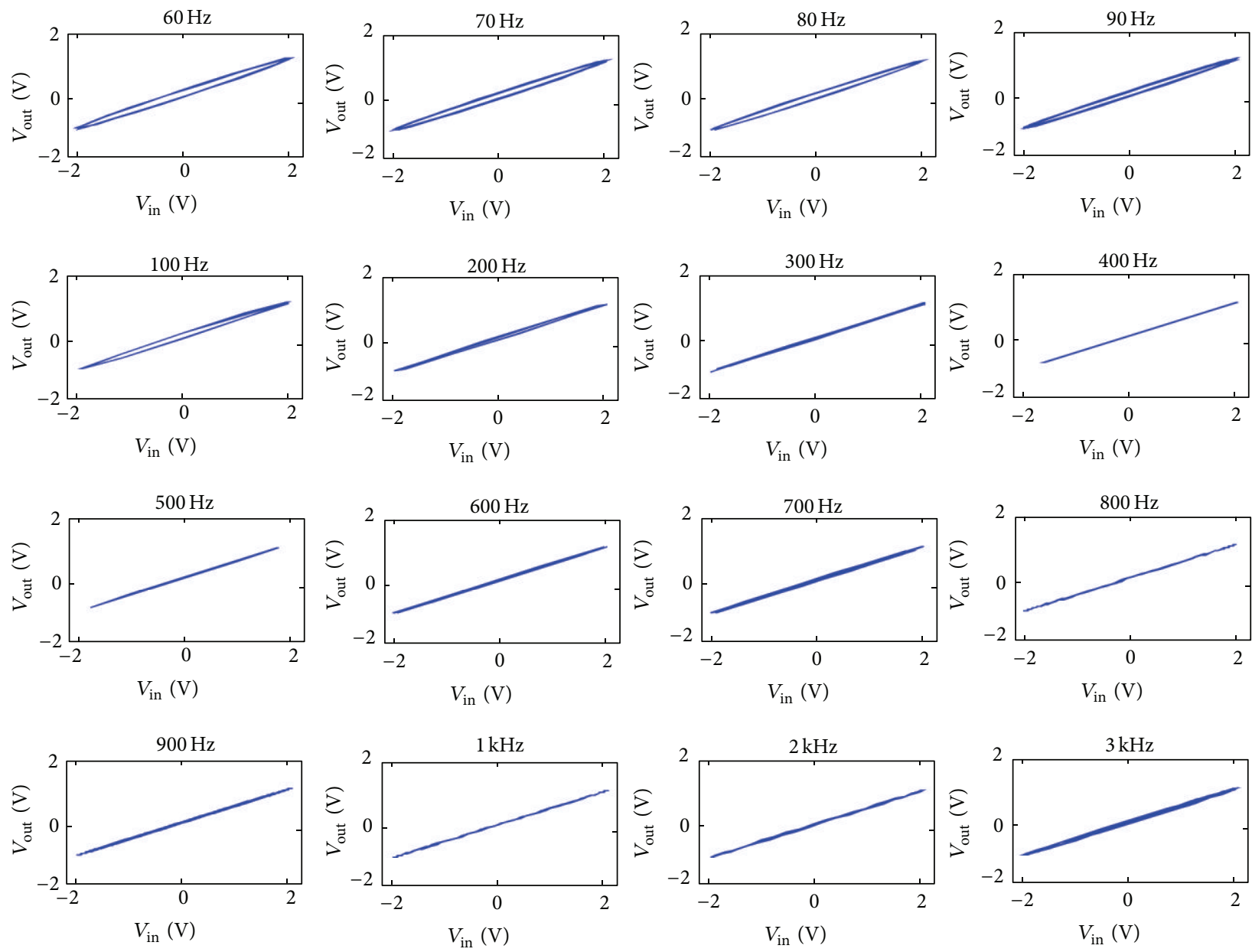

FigURE 7: Lissajous curves on measured input $\left(V_{\text {in }}\right)$ and output $\left(V_{\text {out }}\right)$ voltages in the frequency range between $60 \mathrm{~Hz}$ and $3 \mathrm{kHz}$ for the device $\mathrm{IPMC}_{\text {AbsT-5 h }}$.

4.3. IPMC Frequency Analysis. The diagrams in terms of module and phase of the ratio between $V_{\text {out }}$ and $V_{\text {in }}$ signals at each frequency have been obtained by using the experimental data in the frequencies range between $10 \mathrm{mHz}$ and $10 \mathrm{kHz}$ for both of the devices IPMC AbsT-5 $\mathrm{h}$ and IPMC $\mathrm{AbsT-20} \mathrm{h}_{\text {as shown }}$ in Figures 10 and 11.

Given the conclusion on linearity assessed by the Lissajous curves (Section 4.2), such curves can be considered as the system's frequency responses in terms of Bode diagrams of the transfer function $G_{\text {IPMC }}(s)$ in the linearity range from $1 \mathrm{~Hz}$ to $10 \mathrm{kHz}$.

In such a range, it was observed that both IPMC devices show a fractional-order behavior in a limited span of frequencies where the module of the Bode diagrams presents a slope equal to $m * 20 \mathrm{db} /$ decade, and the phase presents a lag equal to $m * 90^{\circ}$, and where $m$ is a real number conceivable as the fractional-order exponent.

4.3.1. IPMC $C_{A b s T-5 h}$ : IPMC Device with $5 h$ of Absorption Time. The IPMC with $5 \mathrm{~h}$ of absorption time has shown an average slope in the module diagram of $1 \mathrm{~dB}$ per decade in the frequency range between $1 \mathrm{~Hz}$ to $100 \mathrm{~Hz}$, determining $m=0.05$ as clear in Figure 10(a). The phase diagram in Figure $10(\mathrm{~b})$, showed an average phase of $-4.5^{\circ}$ in the same frequency range being coherent with the fractional exponent related to the modules $-m * 90^{\circ}=-4.5^{\circ}$. In conclusion, the IPMC $_{\text {AbsT- } 5 \mathrm{~h}}$ device has shown a fractional-order dynamics in the frequency between $1 \mathrm{~Hz}$ and $100 \mathrm{~Hz}$ with a fractional exponent value of $m=0.05$.

4.3.2. IPMC $C_{A b s T-20 h}$ : IPMC Device with $20 \mathrm{~h}$ of Absorption Time. The IPMC with $20 \mathrm{~h}$ of absorption time showed an average slope in the module diagram of $6 \mathrm{~dB}$ per decade in the frequency range between $1 \mathrm{~Hz}$ and $100 \mathrm{~Hz}$, determining $m=$ 0.3 as clear in Figure 11(a). The phase diagram in Figure 11(b) showed an average phase of $-27^{\circ}$ in the frequency range between $1 \mathrm{~Hz}$ and $10 \mathrm{~Hz}$ showing, in that range, coherence with the fractional exponent related to the modules $-m *$ $90^{\circ}=27^{\circ}$. To conclude, the IPMC AbsT-20h $_{\text {device has shown }}$ a fractional-order dynamics in the frequency between $1 \mathrm{~Hz}$ and $10 \mathrm{~Hz}$ with a fractional exponent value of $m=0.3$. 

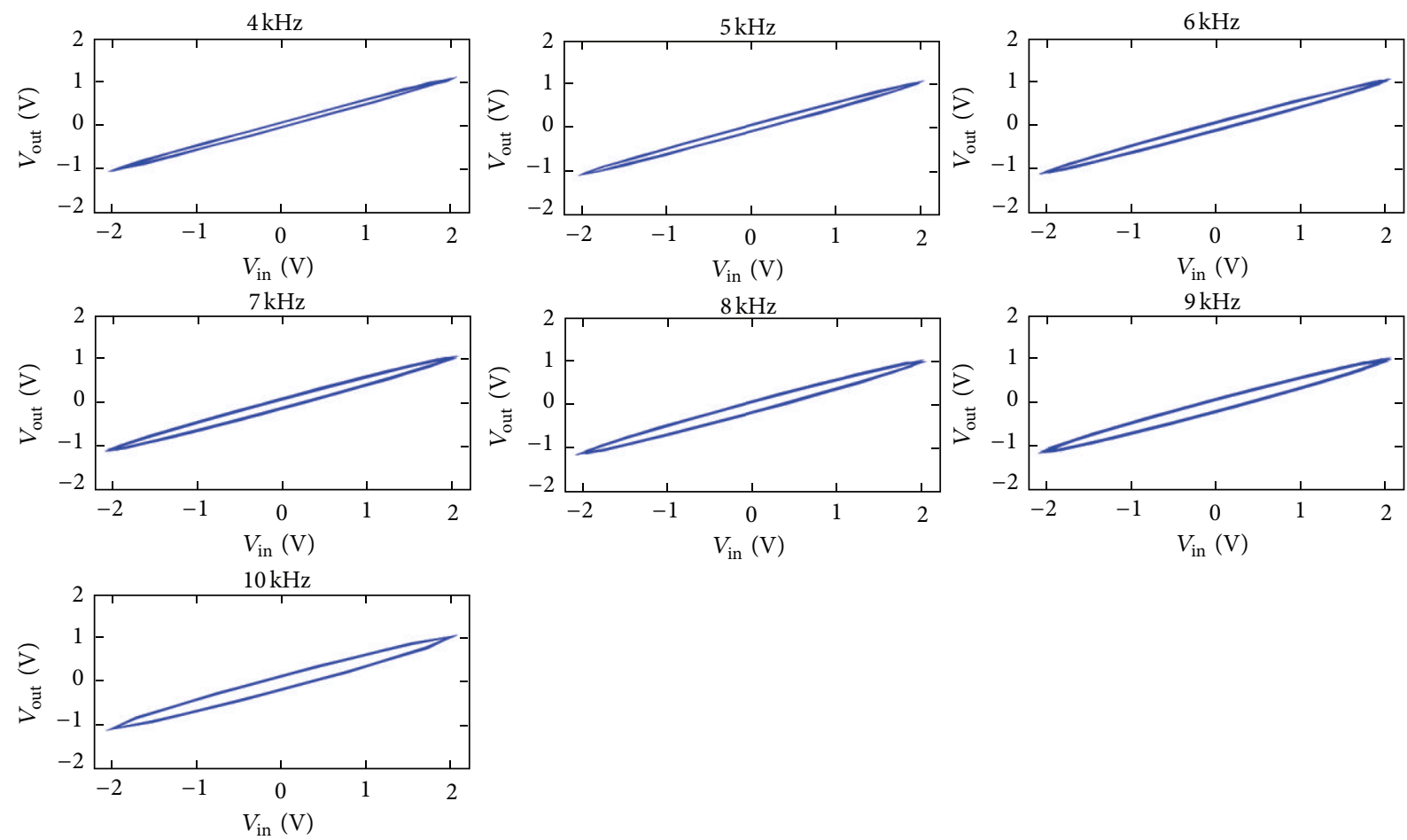

Figure 8: Lissajous curves on measured input $\left(V_{\text {in }}\right)$ and output $\left(V_{\text {out }}\right)$ voltages in the frequency range between $4 \mathrm{kHz}$ and $10 \mathrm{kHz}$ for the device IPMC $\mathrm{AbsT}_{\mathrm{Ab}-5 \mathrm{~h}}$.

\section{Discussion and Conclusion}

This work represents a proof of concept demonstrating the possibility of implementing a compact fractional-order element (FOE) by using a ionic polymer metal composite (IPMC) material.

Previous authors' works, modeling IPMC transducers as fractional-order systems, and the literature evidence, showing that the interaction between ionic phenomena and fractal landscapes is at the bases for FOE working principle, suggested the IPMC as a compact solution for FOEs implementation overcoming dimension issues and suitable for controlling fractional-order exponent values and bandwidth via fabrication processes.

A brand new setup of IPMC device in a fixed sandwich configuration allows neglecting its electromechanical properties and allows focusing on the electrical behavior. Two different IPMCs with different platinum absorption times $(5 \mathrm{~h}$ and $20 \mathrm{~h}$ ) were realized in order to verify how the fabrication parameters affect the electrical dynamics of IPMC. A series of measurement in the frequency domain were therefore performed in order to characterize the behavior of each membrane for a fixed amplitude of the input voltage with the objective of searching experimental evidence of fractionalorder dynamics.

A preliminary linearity study was carried out by analyzing the elliptic shape of the Lissajous curves. It allowed identifying the frequency range between $1 \mathrm{~Hz}$ and $10 \mathrm{KHz}$ where both IPMC devices can be approximated as linear.
The Bode curves of the ratio between the output voltage and the input voltage were considered in the linearity frequency range. They allowed to determine the frequency band where the module and the phase curves are coherent to a fractionalorder dynamics.

In particular, the IPMC with $5 \mathrm{~h}$ of absorption time showed a fractional-order dynamics in the frequency band between $1 \mathrm{~Hz}$ and $100 \mathrm{~Hz}$ with a fractional exponent value of 0.05 , while the IPMC device with $20 \mathrm{~h}$ of absorption time showed fractional-order dynamics in the range between $1 \mathrm{~Hz}$ and $10 \mathrm{~Hz}$ with a fractional exponent value of 0.3 . It is worth noticing that the FOE bandwidth decreases with the increase of the absorption time, while the fractional-order exponent value increases with it.

Such results allow us to take the first steps toward a simplified model of IPMC as a compact electronic FOE with a view of defining the relationship between fabrication parameters, such as the absorption time, the fractional exponent value, and the bandwidth. This concept opens the way for investigation in IPMC FOEs specifics control by other fabrication parameters such as geometry, dispersive agent concentration, solvent, and making it a flexible FOE device suitable for control systems and electronic applications.

\section{Conflict of Interests}

The authors declare that they have no conflict of interests in the research. 


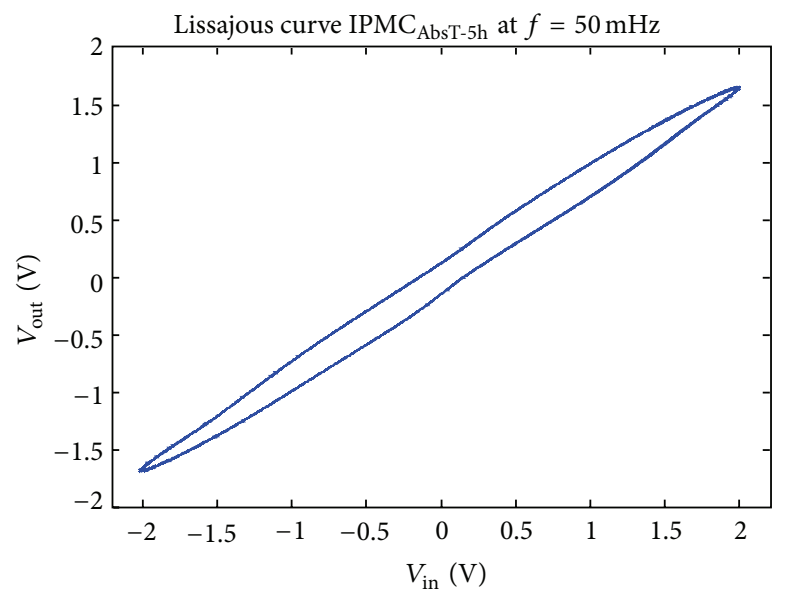

(a)

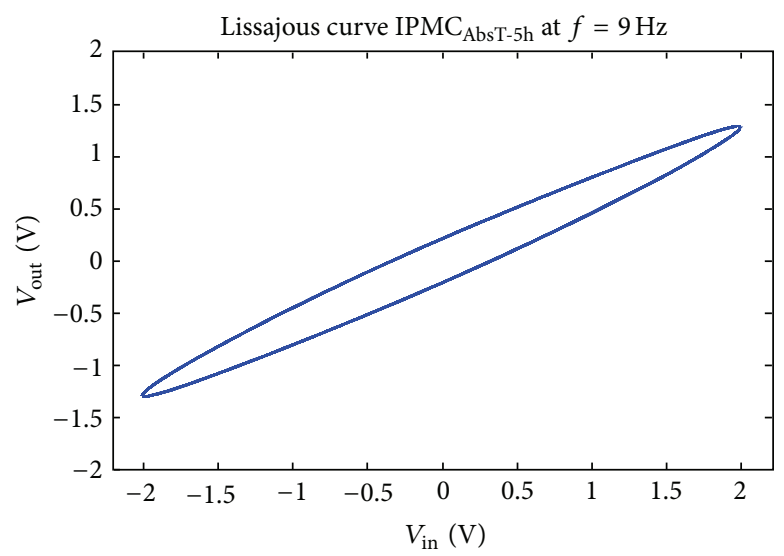

(c)

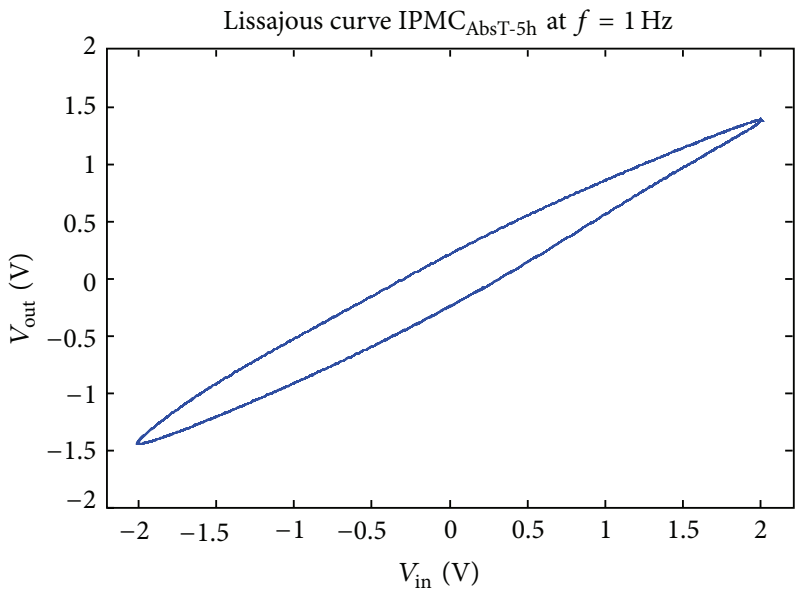

(b)

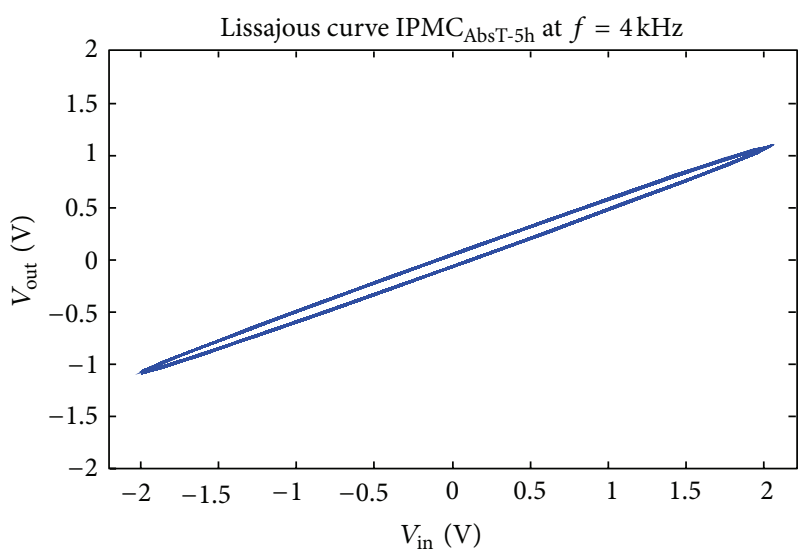

(d)

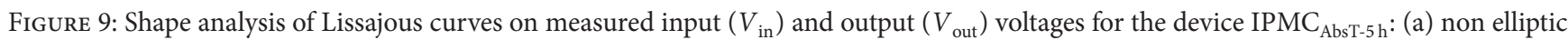
shape at $f=50 \mathrm{mHz}$, (b) transition between nonelliptic and elliptic shapes at $f=1 \mathrm{~Hz}$, (c) elliptic shape at $f=9 \mathrm{~Hz}$, (d) elliptic shape at $f=4 \mathrm{kHz}$.

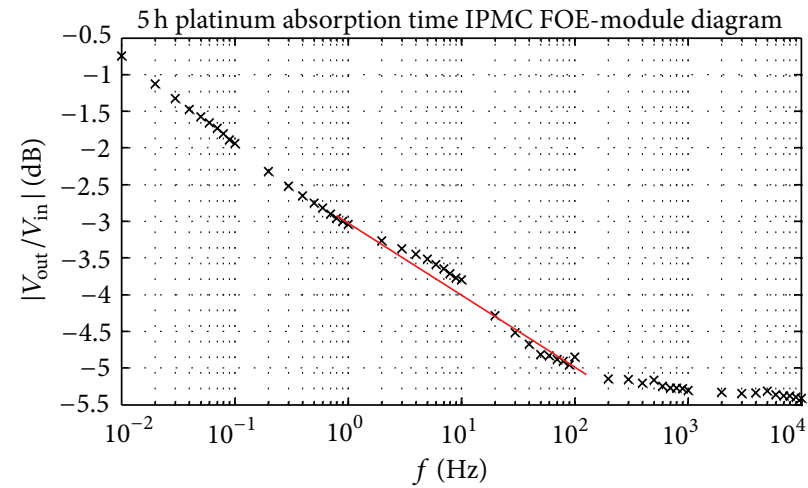

(a)

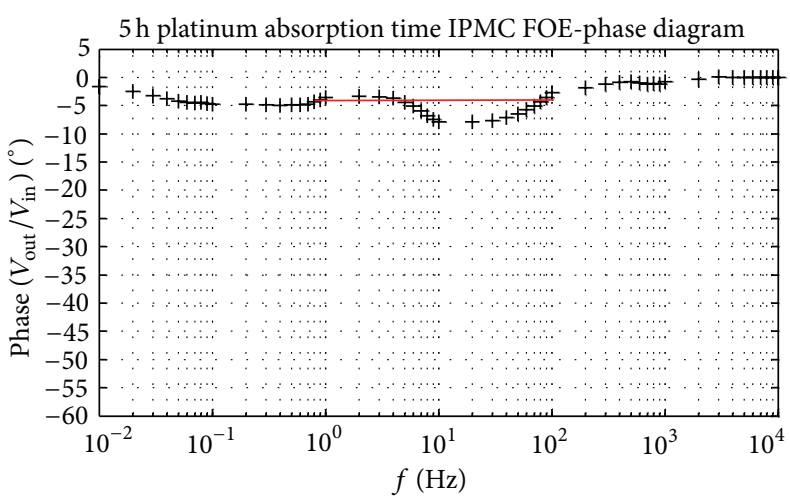

(b)

Figure 10: (a) Module and (b) phase diagrams of the ratio $V_{\text {out }} / V_{\text {in }}$ in the complete experimental frequencies range: $10 \mathrm{mHz}$ to $10 \mathrm{kHz}$ for the IPMC device at $5 \mathrm{~h}$ absorption time. 


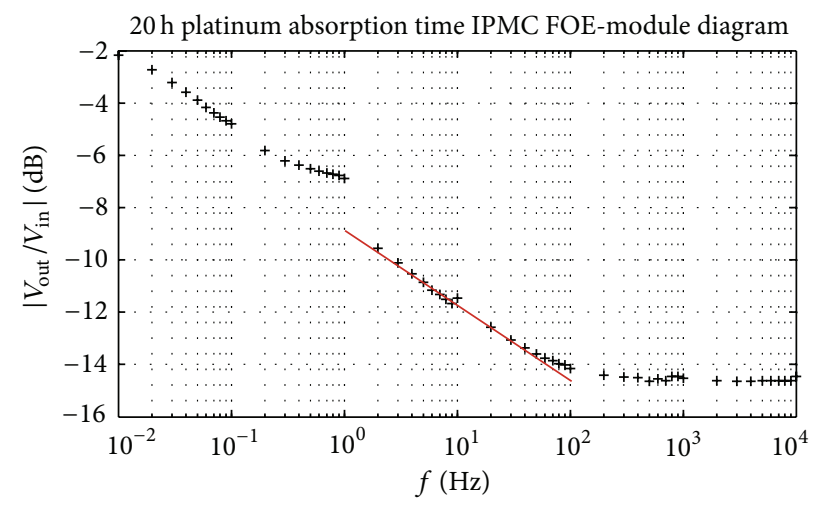

(a)

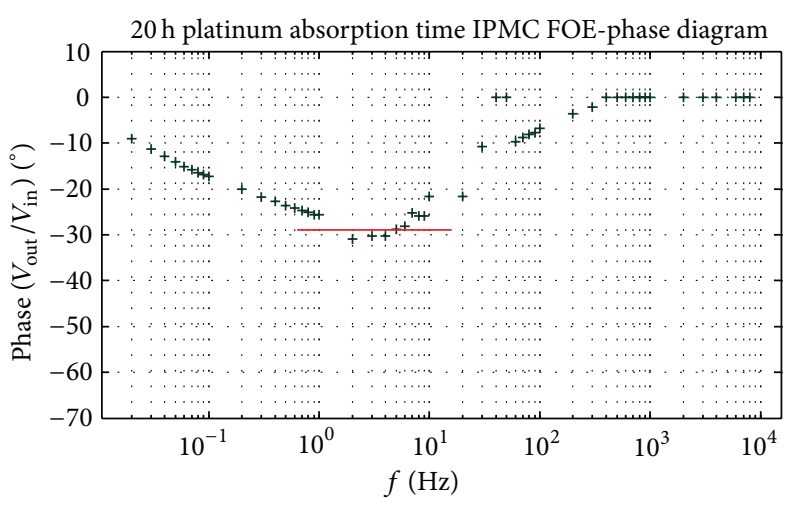

(b)

Figure 11: (a) Module and (b) phase diagrams of the ratio $V_{\text {out }} / V_{\text {in }}$ in the complete experimental frequencies range: $10 \mathrm{mHz}$ to $10 \mathrm{kHz}$ for the IPMC device at $20 \mathrm{~h}$ absorption time.

\section{Acknowledgments}

This work has been partially supported by the Italian Ministry of University and Research (MIUR) under PRIN projects "Noninteger-order Systems in Modeling and Control", Grant no. 2009F4NZJP, and has been developed within the FP7 project SMAC (SMArt systems Co-design), G.A. no. 288827, at UNICT.

\section{References}

[1] M. Shahinpoor and K. J. Kim, "Ionic polymer-metal composites: I. Fundamentals," Smart Materials and Structures, vol. 10, no. 4, pp. 819-833, 2001.

[2] M. Shahinpoor, Y. Bar-Cohen, J. O. Simpson, and J. Smith, "Ionic polymer-metal composites (IPMCs) as biomimetic sensors, actuators and artificial muscles-a review," Smart Materials and Structures, vol. 7, no. 6, pp. R15-R30, 1998.

[3] M. Shahinpoor and K. J. Kim, "Ionic polymer-metal composites: IV. Industrial and medical applications," Smart Materials and Structures, vol. 14, no. 1, pp. 197-214, 2005.

[4] W.-S. Chu, K. T. Lee, S.-H. Song et al., "Review of biomimetic underwater robots using smart actuators," International Journal of Precision Engineering and Manufacturing, vol. 13, no. 7, pp. 1281-1292, 2012.

[5] D. Pugal, K. Jung, A. Aabloo, and K. J. Kim, "Ionic polymermetal composite mechanoelectrical transduction: review and perspectives," Polymer International, vol. 59, no. 3, pp. 279-289, 2010.

[6] K. M. Newbury and D. J. Leo, "Linear electromechanical model of ionic polymer transducers-part I: model development," Journal of Intelligent Material Systems and Structures, vol. 14, no. 6, pp. 333-342, 2003.

[7] R. Kanno, A. Kurata, S. Tadokoro, T. Takamori, and K. Oguro, "Characteristics and modeling of ICPF actuator," in Proceedings of the Japan-USA Symposium on Flexible Automation, pp. 219225,1994

[8] C. Bonomo, L. Fortuna, P. Giannone, S. Graziani, and S. Strazzeri, "A nonlinear model for ionic polymer metal composites as actuators," Smart Materials and Structures, vol. 16, no. 1, pp. 1-12, 2007.
[9] P. Brunetto, L. Fortuna, S. Graziani, and S. Strazzeri, "A model of ionic polymer-metal composite actuators in underwater operations," Smart Materials and Structures, vol. 17, no. 2, Article ID 025029, 12 pages, 2008.

[10] C. Bonomo, L. Fortuna, P. Giannone, S. Graziani, and S. Strazzeri, "A model for ionic polymer metal composites as sensors," Smart Materials and Structures, vol. 15, no. 3, pp. 749758, 2006.

[11] D. Pugal, H. Kasemägi, K. J. Kim, M. Kruusmaa, and A. Aabloo, "Finite element simulations of the bending of the IPMC sheet," in Electroactive Polymer Actuators and Devices (EAPAD '07), Proceedings of SPIE, San Diego, Calif, USA, March 2007.

[12] R. Caponetto, V. De Luca, S. Graziani, F. Sapuppo, and E. Umana, "A multi-physics model of an IPMC actuator in the electrical, chemical, mechanical and thermal domains," in Proceedings of the International Conference on Synthesis, Modeling, Analysis and Simulation Methods and Applications to Circuit Design (SMACD '12), Seville, Spain, September 2012.

[13] R. Caponetto, G. Dongola, L. Fortuna, S. Graziani, and S. Strazzeri, "A fractional model for IPMC actuators," in Proceedings of IEEE International Instrumentation and Measurement Technology Conference, pp. 2103-2107, Victoria, Canada, May 2008.

[14] L. Chang, H. Chen, and Z. Zhu, "A structure model for Ionic polymer-metal composite (IPMC)," in Electroactive Polymer Actuators and Devices (EAPAD '12), vol. 8340 of Proceedings of SPIE, April 2012.

[15] S. C. D. Roy, "On the realization of a constant-argument immittance or fractional operator," IEEE Transactions on Circuit Theory, vol. 14, no. 3, pp. 264-274, 1967.

[16] G. W. Bohannan, "Analog realization of a fractional control element-revisited," 2002.

[17] H. Sheng, H. G. Sun, C. Coopmans, Y. Q. Chen, and G. W. Bohannan, "A physical experimental study of variable-order fractional integrator and differentiator," European Physical Journal: Special Topics, vol. 193, no. 1, pp. 93-104, 2011.

[18] T. C. Haba, G. Ablart, T. Camps, and F. Olivie, "Influence of the electrical parameters on the input impedance of a fractal structure realised on silicon," Chaos, Solitons \& Fractals, vol. 24, no. 2, pp. 479-490, 2005. 
[19] G. W. Bohannan, "Analog fractional order controller in temperature and motor control applications," Journal of Vibration and Control, vol. 14, no. 9-10, pp. 1487-1498, 2008.

[20] I. S. Jesus and J. A. Tenreiro MacHado, "Development of fractional order capacitors based on electrolyte processes," Nonlinear Dynamics, vol. 56, no. 1-2, pp. 45-55, 2009.

[21] K. Biswas, S. Sen, and P. K. Dutta, "Realization of a constant phase element and its performance study in a differentiator circuit," IEEE Transactions on Circuits and Systems II, vol. 53, no. 9, pp. 802-806, 2006.

[22] K. Biswas, Studies on design, development and performance analysis of capacitive type sensors [Ph.D. dissertation], Dep. Elect. Eng., IIT Kharagpur, Kharagpur, India, 2006.

[23] M. S. Krishna, S. Das, K. Biswas, and B. Goswami, "Characterization of a fractional order element realized by dipping a capacitive type probe in polarizable medium," in Proceedings of the Lisbon Symposium on Fractional Signals and Systems, Lisbon, Portugal, November 2009.

[24] "Aldrich Technical Information Bulletin AL-163," Nafion Resins.

[25] K. B. Oldham and J. Spanier, The Fractional Calculus: Theory and Applications of Differentiation and Integration to Arbitrary Order, Dover Books on Mathematics, 2006.

[26] I. Podlubny, Fractional Differential Equations, vol. 198 of Mathematics in Science and Engineering, Academic Press, San Diego, Calif, USA, 1999.

[27] R. Caponetto, G. Dongola, L. Fortuna, and I. Petras, "Fractional order systems: modelling and control applications," in Nonlinear Science, Series A, vol. 72, World Scientific, Singapore, 2010.

[28] Z. Jiao, Y. Chen, and I. Podlubny, Distributed-Order Dynamic Systems, Springer, New York, NY, USA, 2012. 


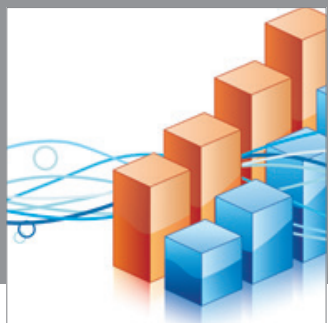

Advances in

Operations Research

mansans

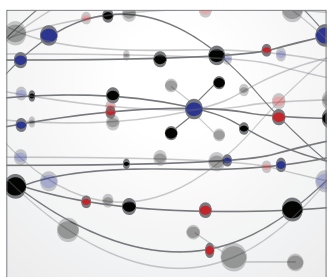

The Scientific World Journal
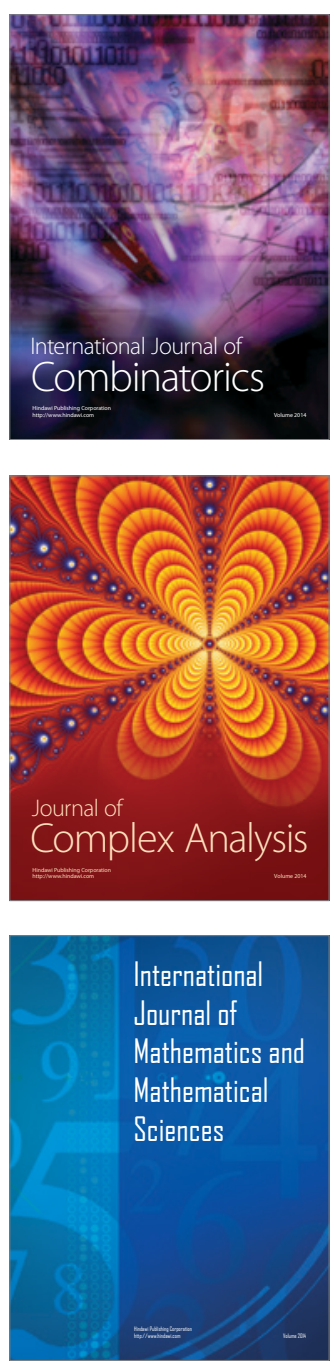
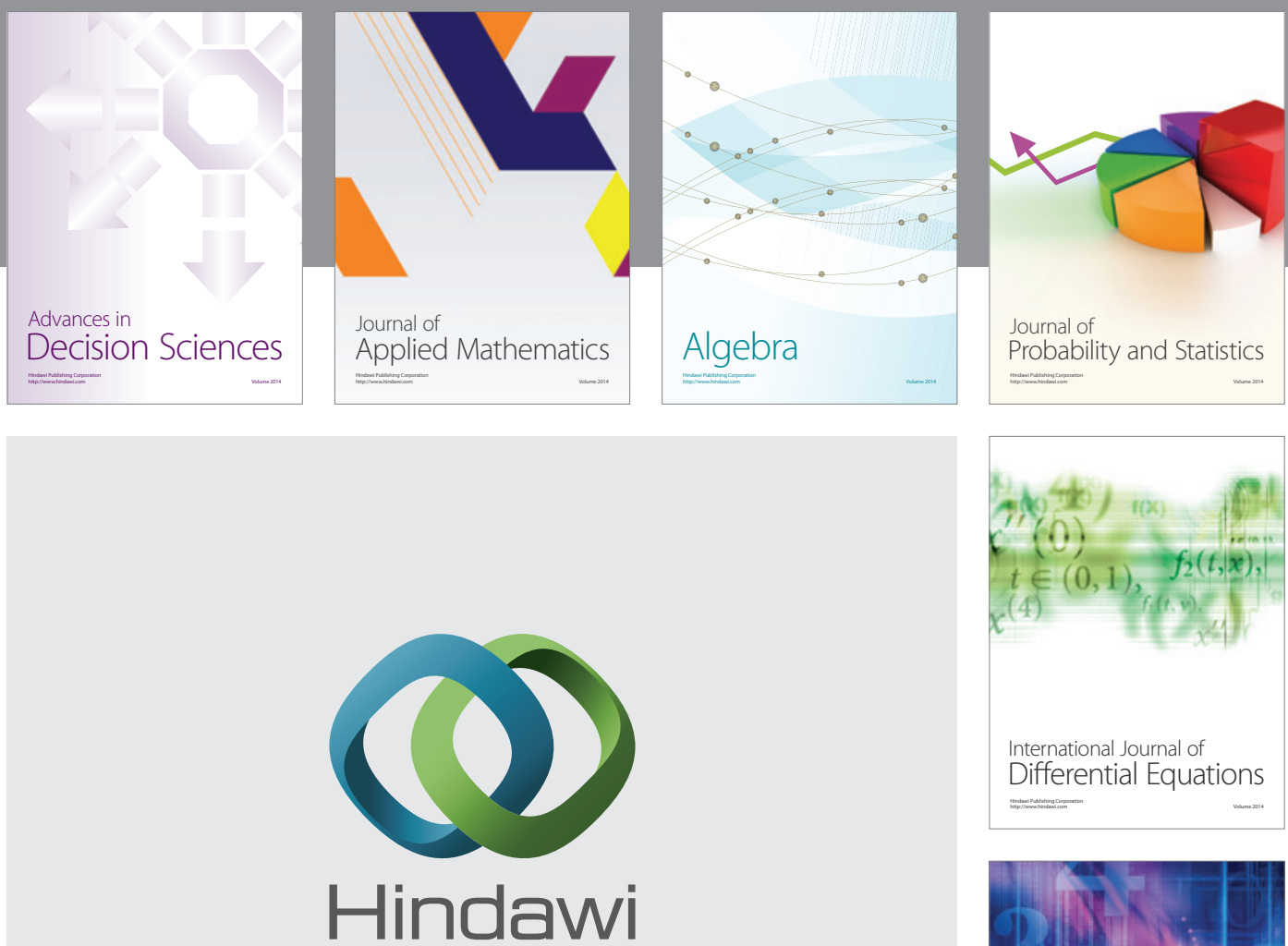

Submit your manuscripts at http://www.hindawi.com
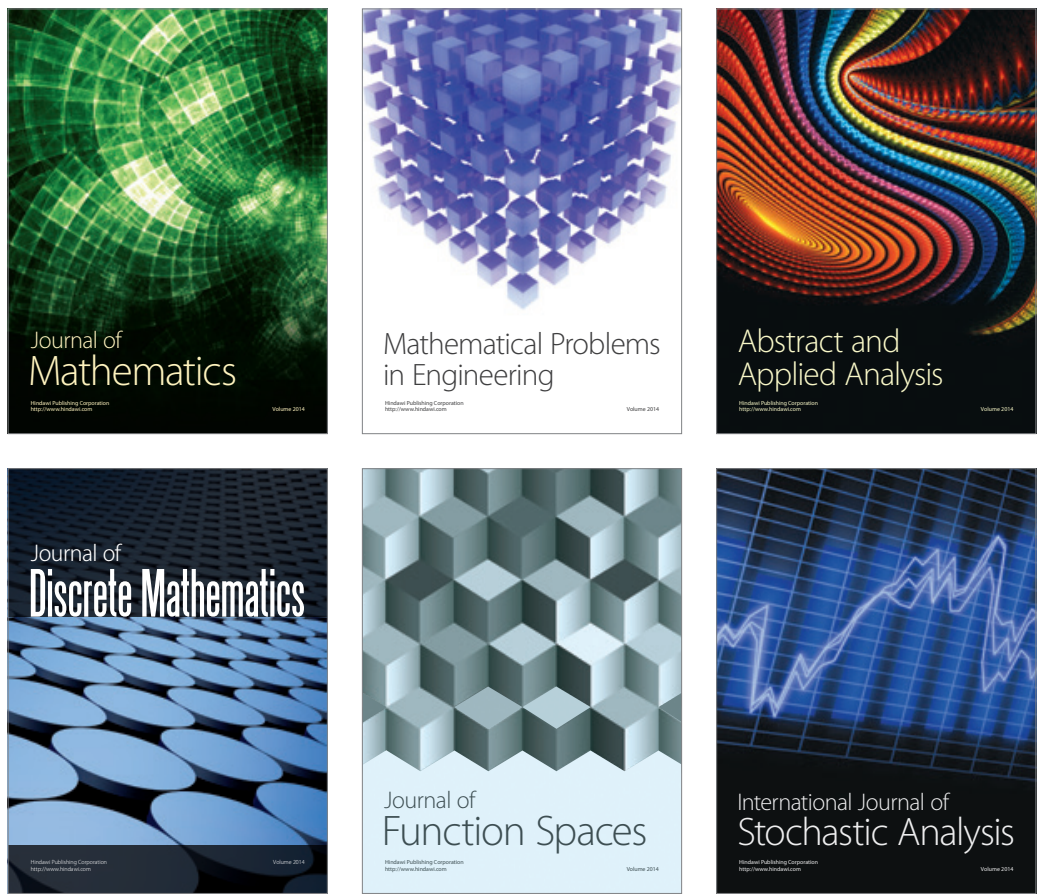

Journal of

Function Spaces

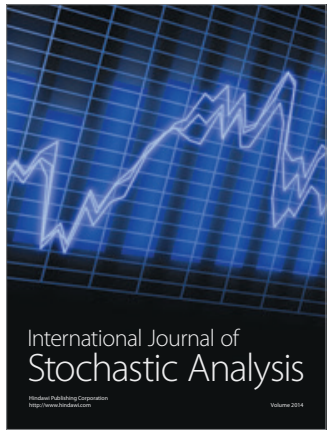

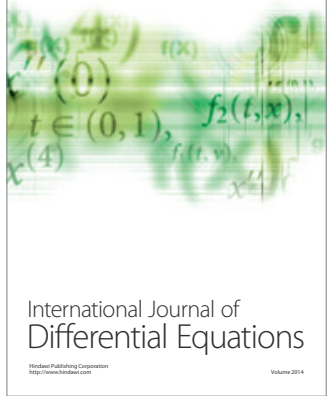
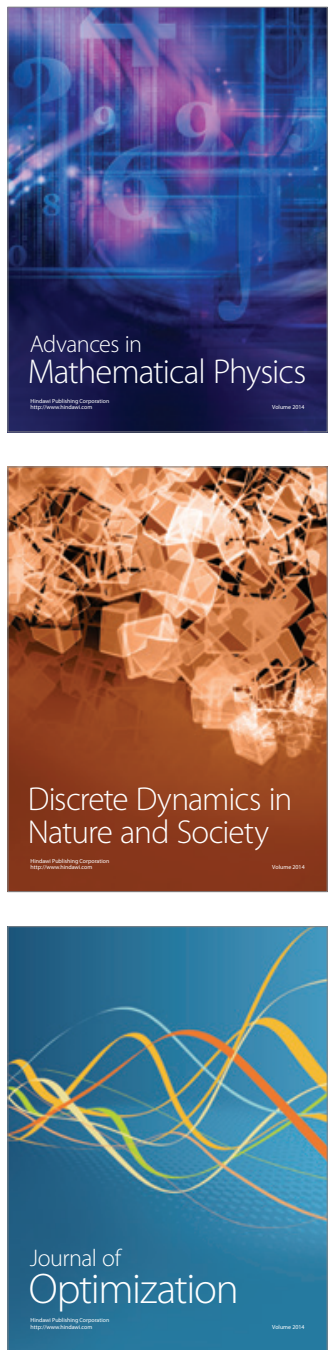